\title{
Review of the direct derivation method: quantitative phase analysis with observed intensities and chemical composition data
}

\author{
Hideo Toraya $\mathbb{D}^{*}$ \\ Rigaku Corporation, Matsubara, Akishima, Tokyo 196-8666, Japan
}

(Received 14 April 2021; accepted 18 May 2021)

\begin{abstract}
The direct derivation (DD) method is a technique for quantitative phase analysis (QPA). It can be characterized by the use of the total sums of scattered/diffracted intensities from individual components as the observed data. The crystal structure parameters are required when we calculate the intensities of reflections or diffraction patterns. Intensity can, however, be calculated only with the chemical composition data if it is not of individual reflections but of a total sum of diffracted/scattered intensities for that material. Furthermore, it can be given in a form of the scattered intensity per unit weight. Therefore, we can calculate the weight proportion of a component material by dividing the total sum of observed scattered/diffracted intensities by the scattered intensity per unit weight. The chemical composition data of samples under investigation are known in almost all cases at the stage of QPA. Thus, a technical problem is how to separate the observed diffraction pattern of a mixture into individual component patterns. Various pattern decomposition techniques currently available can be used for separating the pattern of a mixture. In this report, the theoretical background of the DD method and various techniques for pattern decompositions are reviewed along with the examples of applications. () The Author(s), 2021. Published by Cambridge University Press on behalf of International Centre for Diffraction Data. This is an Open Access article, distributed under the terms of the Creative Commons Attribution licence (https://creativecommons.org/licenses/by/4.0/), which permits unrestricted re-use, distribution, and reproduction in any medium, provided the original work is properly cited. [doi:10.1017/S0885715621000373]
\end{abstract}

Key words: direct derivation method, quantitative phase analysis, intensity-composition formula, $\mathrm{X}$-ray powder diffraction, chemical composition data

\section{INTRODUCTION}

Currently used methods for quantitative phase analysis (QPA) can be classified into three categories according to the form of observed X-ray intensity data used in deriving the weight fractions of individual components in a mixture. Methods in the first category use single peak intensities as the observed data. The internal standard method (Alexander and Klug, 1948), the method of standard addition (Lennox, 1957), the reference intensity ratio (RIR) method (Chung, 1974a, 1974b; Hubbard et al., 1976), etc. can be grouped into this category. In the second category, the calculated/ observed powder patterns are used for modeling the diffraction patterns of individual components in whole-powderpattern fitting (WPPF). The Rietveld method for QPA (Rietveld, 1969; Werner et al., 1979) and the full-pattern fitting methods using single-phase observed diffraction patterns (Smith et al., 1987; Chipera and Bish, 2002) can be included into this category. The direct derivation (DD) method, which uses the total sums of intensities for individual components can be classified into the third category (Toraya, 2016; Toraya and Omote, 2019). Readers of this article may be familiar with methods for QPA in the former two categories. The internal standard method, proposed more than 70 years

\footnotetext{
*Author to whom correspondence should be addressed. Electronic mail:
} toraya@ rigaku.co.jp ago, is still widely used for the quantification of a target component in a mixture. The Rietveld QPA, proposed 40 years ago (Werner et al., 1979), has been used as an indispensable tool for materials characterization. The method in the third category, the DD method, is a new one, proposed just 4 years ago, and many readers will have simple questions why and how to use the total sums of intensities for QPA.

In applying the single-peak intensity methods, intensity ratios to reference materials are required to be predetermined experimentally as in the calibration curve method as well as the RIR method or by calculation as in the direct comparison method (Averbach and Cohen, 1948). In the Rietveld QPA, the crystal structure parameters are required to calculate the individual component patterns. The crystal structure parameters are necessary when we calculate the intensity of a reflection or a diffraction pattern. If the intensity is not of individual reflections but of a total sum of diffracted/scattered intensities from the material, however, the intensity can be calculated only with the chemical composition data: the chemical formula weight and the numbers of electrons belonging to atoms in the chemical formula unit. This is a reason why the DD method uses the total sums of intensities as observed data together with the chemical composition data to derive the weight fractions of individual components. QPA cannot be conducted by the RIR method or Rietveld QPA when RIR values or crystal structure parameters are missing even for just one component in the target mixture, while the 
chemical compositional information will in almost all cases be available.

As will be described later, the DD method itself is based on a simple principle. There are, however, various ways to obtain the total sums of diffracted/scattered intensities from the target mixture pattern, and they have been reported in several papers (Toraya, 2016, 2017, 2018, 2019, 2020). In this report, it is tried to review comprehensively the theoretical background of the DD method and the techniques for the pattern decomposition. Some examples of applications are also given.

\section{THEORY}

\section{A. Total sum of intensities}

Theory of the DD method can be started from the intensity formula for powder diffraction (James, 1967). For the $j$ th reflection from a thick slab of powdered specimen in the Bragg-Brentano (BB) geometry, the integrated intensity, $I_{j}$, can be calculated by

$$
I_{j}=C \frac{1}{\mu} \frac{V}{U^{2}} m_{j}\left|F_{j}\right|^{2} G_{j}^{-1}
$$

where $C$ represents the proportional constant including the wavelength of incident X-rays, the mass and charge of electron, the velocity of light in free space, the width of a receiving slit, and the goniometer radius, $\mu$ is the linear absorption coefficient, $V$ is the irradiated volume of specimen, $U$ is the unit-cell volume, $m_{j}$ is the multiplicity of reflection, $F_{j}$ is the structure factor, and $G_{j}^{-1}$ represents the Lorentzpolarization factor and the geometrical factor. In the present case based on the BB geometry, $G_{j}^{-1}$ can be given by $G_{j}^{-1}=\left(1+\cos ^{2} 2 \theta_{j}\right) /\left(2 \sin \theta_{j} \sin 2 \theta_{j}\right)$, when no monochromator on both incident- and diffracted-beam sides is assumed. By multiplying both terms of Eq. (1) by $G_{j}$ and summing up them, we obtain

$$
\sum_{j=1}^{N} I_{j} G_{j}=C \frac{1}{\mu} \frac{V}{U}\left(\frac{1}{U} \sum_{j=1}^{N} m_{j}\left|F_{j}\right|^{2}\right)
$$

where $N$ is the number of reflections in a wide $2 \theta$-range defined by $\left[2 \theta_{\mathrm{L}}, 2 \theta_{\mathrm{H}}\right]$. In Eq. (2), $U^{-1} \sum m_{j}\left|F_{j}\right|^{2}$ is identical with a peak height at the origin of the Patterson function, $P$ (0). We often approximate the integrated intensity of a reflection with its peak height. In the same manner but inversely in this case, the peak height, $P(0)$, can be approximated by the integrated value of the peak, which can be given by $Z \sum n_{i}^{2}$, where $Z$ is the number of chemical formula unit in the unit cell, $n_{i}$ is the number of electrons belonging to the $i$ th atom in the chemical formula unit with the total number of atoms $N^{\mathrm{A}}$, and the summation is taken over $N^{\mathrm{A}}$ atoms. Then, we obtain

$$
\sum_{j=1}^{N} I_{j} G_{j} \cong C \frac{1}{\mu} \frac{V}{U}\left(Z \sum_{i=1}^{N^{\mathrm{A}}} n_{i}^{2}\right)
$$

$V / U$ in Eq. (3) represents the number of unit cell in the volume $V$, and it can equivalently be represented by $W / Z M$, where $W$ is the weight of a specimen with the volume $V$ and
$M$ is the chemical formula weight.' By replacing $V / U$ with the $W / Z M$, we obtain

$$
\sum_{j=1}^{N} I_{j} G_{j} \cong C \frac{1}{\mu} \frac{W}{M} \sum_{i=1}^{N^{\mathrm{A}}} n_{i}^{2}
$$

The $V$ in Eq. (3) can also be represented by $V=W(Z M / U)^{-1}$, where $Z M / U$ is the density of the material, and it derives the same expression as Eq. (4). Equation (4) was first derived by using the approximation as described above: the relationship between the height and the integrated value of the peak at the origin of the Patterson function (Toraya, 2016). The expression, which is identical to that of Eq. (4), had also been derived in a more rigorous form, starting from the Debye equation, by integrating over the reciprocal space the scattered intensity from an assemblage of atoms at arbitrary positions [Eq. (15) in Toraya and Omote (2019)]. Theoretical derivation by Toraya and Omote (2019) for the random assemblage of atoms can also be applied to the ordered arrangement of atoms. Therefore, Eq. (4) is considered to be valid for both crystalline and noncrystalline materials.

In Eq. (4), $\sum n_{i}^{2}$ represents the total scattered intensity from atoms in the chemical formula unit. Thus, $\sum n_{i}^{2} / M$ represents the scattered intensity per unit weight. When the $\sum n_{i}^{2} / M$ is multiplied by $W$, the product should give the total intensity from the specimen. Since $W / M$ represents the number of chemical formula units in the specimen with the weight $W$, Eq. (4) can alternatively be interpreted as representing (the number of chemical formula unit) $\times$ (the scattered intensity from the chemical formula unit). Both interpretations give the same quantity of the total scattered intensity from the specimen, and they are consistent with the $\sum I_{j} G_{j}$ on the lefthand side of Eq. (4).

\section{B. The intensity-composition formula}

Let us consider a $K$-component mixture and introduce two parameters, $S_{k}$ and $a_{k}^{-1}$, where the subscript $k$ represents the $k$ th component. The $S_{k}$ and $a_{k}^{-1}$ are given by

$$
\begin{gathered}
S_{k}=\sum_{j=1}^{N} I_{k j} G_{j} \\
a_{k}^{-1}=\frac{1}{M_{k}} \sum_{i=1}^{N_{k}^{\mathrm{A}}} n_{k i}^{2}
\end{gathered}
$$

$S_{k}$ represents the total sum of intensities corrected for the factor $G_{j}^{-1}$, and it is an observable quantity. $a_{k}^{-1}$ represents the scattered intensity per unit weight, and its magnitude can be calculated only with the chemical composition data: the chemical formula weight and the numbers of electrons. Equation (6) does not include any crystallographic parameters like $Z$ or $U$ of component materials, and the input data required for calculating $a_{k}^{-1}$

\footnotetext{
${ }^{1}$ The relation between the $V / U$ and $W / Z M$ can more precisely be expressed by $V / U=0.602 \times(W / Z M)$, where a factor 0.602 appears for keeping the consistency in units of gram, cubic angstrom, etc. This expression can also be applied to represent the density $(d)$ by $d=Z M /(0.602 \times U)$.
} 
are purely chemical. Equation (4) can be expressed by

$$
W_{k}=\frac{\mu}{C} \frac{S_{k}}{a_{k}^{-1}}=\frac{\mu}{C} a_{k} S_{k}
$$

In QPA, what we would like to find are the relative weight ratios of the individual components. Therefore, under the normalization condition, the weight fraction for the $k$ th component, $w_{k}$, can be calculated by

$$
w_{k}=\frac{W_{k}}{W}=W_{k}\left(\sum_{k^{\prime}=1}^{K} W_{k^{\prime}}\right)^{-1}
$$

As long as intensities of individual components in a mixture, measured in a single scan, are compared, a single value of the linear absorption coefficient $\mu$ is shared with all components. Thus, $\mu$ and $C$ in Eq. (7) are canceled out, when Eq. (7) is substituted into Eq. (8). Then, the $w_{k}$ is given by

$$
w_{k}=a_{k} S_{k}\left(\sum_{k^{\prime}=1}^{K} a_{k^{\prime}} S_{k^{\prime}}\right)^{-1}
$$

Equation (9) is called "the intensity-composition (IC) formula" as will be understood from two parameters $S_{k}$ and $a_{k}$ (Toraya, 2016).

\section{Observed intensity datasets for individual components}

As described above, the total sums of intensities $\left(S_{k}\right)$ for individual components are used as observed data in the IC formula. Various techniques of the powder pattern fitting can selectively be used for deriving $\left\{S_{k}\right\}$ for each sample. Fitting functions used in these techniques are currently classified into four types, A, B, C, and $\mathrm{C}_{2}$, and they are summarized in Table I (Toraya, 2019).

A powder diffraction pattern of a mixture, $y(2 \theta)$, is the superposition of individual component patterns, $y(2 \theta)_{k}$, and it can be represented by

$$
\mathrm{y}(2 \theta)=y(2 \theta)_{\mathrm{BG}}+\sum_{k=1}^{K} y(2 \theta)_{k}
$$

where $y(2 \theta)_{\mathrm{BG}}$ is the background (BG) intensity, and some analytical functions, such as polynomials, can be used for modeling the BG. The type-A function is the fitting function widely used in the Pawley or Le Bail method for whole-powder-pattern decomposition (WPPD; Pawley, 1981; Le Bail et al., 1988). It can be expressed by $y(2 \theta)_{k}=\sum_{j} I_{k j} P(2 \theta)_{k j}$, where $P(2 \theta)_{k j}$ is the normalized profile function and $I_{k j}$ are adjustable. Individual profile fitting (IPF) techniques (Taupin, 1973; Parrish et al., 1976) also belong to this group, and they can be used when reflections are sparsely distributed in a mixture pattern.

When strong parameter correlations of $I_{k j}$ are inevitable for heavily overlapping reflections, the type-B function can effectively be used. The type-B function uses a predetermined intensity parameter dataset $\left\{I_{k j}^{\prime}\right\}$. It can be defined by $y(2 \theta)_{k}=S c_{k} \sum_{j} I_{k j}^{\prime} P(2 \theta)_{k j}$, where $S c_{k}$ is the scale parameter, and the $S c_{k}$ is adjusted while $\left\{I_{k j}^{\prime}\right\}$ are fixed at their original values in WPPF (Toraya and Tsusaka, 1995). The intensity dataset $\left\{I_{k j}^{\prime}\right\}$ can be obtained by decomposing a single-phase powder diffraction pattern of the $k$ th component by using the WPPD methods or by calculation from the crystal structure parameters. When the type-B function is used, $S_{k}$ is given by $S_{k}=S c_{k} \sum I_{k j}^{\prime} G_{j}$.

When the pattern decomposition nor the calculation from the crystal structure parameters is difficult for obtaining $\left\{I_{k j}\right\}$ or $\left\{I_{k j}^{\prime}\right\}$ as in the case of clay minerals with staking disorder, the type- $C$ function can effectively be used. The type- $C$ function uses a single-phase observed diffraction pattern after subtracting the BG just as in the full-pattern fitting method by Smith et al. (1987). The profile intensity for the single-phase $k$ th component material is represented by

$$
y(2 \theta)_{k}^{\mathrm{S}}=y(2 \theta)_{\mathrm{BG} \_}^{\prime}+y(2 \theta)_{k}^{\prime}
$$

where $y(2 \theta)_{\mathrm{BG} \_k}^{\prime}$ is the BG intensity, $y(2 \theta)_{k}^{\prime}$ is the peak profile intensity, and a superscript $\mathrm{S}$ is used to denote "single phase". The type-C function is given by $y(2 \theta)_{k}=S c_{k} y(2 \theta)_{k}^{\prime}$, and the scale parameter $S c_{k}$ is adjusted in WPPF. The total sum of intensities can be obtained by

$$
Y_{k}=S c_{k} \int_{2 \theta_{\mathrm{L}}}^{2 \theta_{\mathrm{H}}} y(2 \theta)_{k}^{\prime} G(2 \theta) \mathrm{d}(2 \theta)
$$

where $G(2 \theta)$ is the continuous form of $G_{j}$. Since the definition of $Y_{k}$ by Eq. (12) is different from that of $S_{k}$ by Eq. (5), different symbols $S_{k}$ and $Y_{k}$ are used. However, $S_{k}$ and $Y_{k}$ can easily be verified to be equivalent for a pattern in the same $2 \theta$-range $\left[2 \theta_{\mathrm{L}}, 2 \theta_{\mathrm{H}}\right.$, and the $Y_{k}$ can equally be used in place of $S_{k}$ in Eq. (9) (Toraya, 2018).

When the BG subtraction is technically difficult as in the case of a halo pattern from an amorphous material, the type- $\mathrm{C}_{2}$ function can be used. The type- $C_{2}$ function uses $y(2 \theta)_{k}^{\mathrm{S}}$ for modeling a component pattern without subtracting the BG (Chipera and Bish, 2002), and it is simply defined by $y(2 \theta)_{k}^{\mathrm{BP}}=S c_{k} y(2 \theta)_{k}^{\mathrm{S}}=S c_{k}\left[y(2 \theta)_{\mathrm{BG} \_k}^{\prime}+y(2 \theta)_{k}^{\prime}\right]$, where superscript $\mathrm{BP}$ is used to mean $\mathrm{BG}+$ peak profile intensities. In this case, $S c_{k} y(2 \theta)_{\mathrm{BG} \_k}^{\prime}$ works as a part of the BG model. The

TABLE I. Fitting functions currently used for separating the observed pattern of a mixture into individual component patterns.

\begin{tabular}{lllll}
\hline \hline Function & Methods & Fitting functions & Intensity parameters refined & Output data \\
\hline Type-A & WPPD, IPF & $\sum I_{j k} P(2 \theta)_{j k}$ & $I_{j k}$ & $S_{k}$ \\
Type-B & WPPD, Rietveld & $S c_{k} \sum I_{j k}^{\prime} P(2 \theta)_{j k}$ & $S c_{k}$ & $S_{k}$ \\
Type-C & Full-pattern fitting & $S c_{k} y(2 \theta)_{k}^{\prime}$ & $S c_{k}$ & $Y_{k}$ \\
Type-C ${ }_{2}$ & Full-pattern fitting & $S c_{k} y(2 \theta)_{k}^{\mathrm{S}}$ & $S c_{k}$ & $Y_{k}^{\mathrm{BP}}$ \\
\hline \hline
\end{tabular}

References for individual methods will be found in Section II.C. 
integration of $S c_{k} y(2 \theta)_{k}^{\mathrm{S}} G(2 \theta)$ in the range $\left[2 \theta_{\mathrm{L}}, 2 \theta_{\mathrm{H}}\right]$ is given by

$$
\begin{aligned}
Y_{k}^{\mathrm{BP}}= & S c_{k} \int_{2 \theta_{\mathrm{L}}}^{2 \theta_{\mathrm{H}}} y(2 \theta)_{\mathrm{BG} \_k}^{\prime} G(2 \theta) \mathrm{d}(2 \theta) \\
& +S c_{k} \int_{2 \theta_{\mathrm{L}}}^{2 \theta_{\mathrm{H}}} y(2 \theta)_{k}^{\prime} G(2 \theta) \mathrm{d}(2 \theta)
\end{aligned}
$$

By denoting the first term on the right-hand side of Eq. (13) by $B_{k}, Y_{k}^{\mathrm{BP}}$ can be expressed by $Y_{k}^{\mathrm{BP}}=B_{k}+Y_{k}$. Let us define a ratio of $B_{k}$ to $Y_{k}$ by $R_{k}=B_{k} / Y_{k}$. Then, $Y_{k}$ can be obtained from $Y_{k}^{\mathrm{BP}}$ by

$$
Y_{k}=\frac{Y_{k}^{\mathrm{BP}}}{1+R_{k}}
$$

When the type- $\mathrm{C}_{2}$ function is assigned to modeling the $k$ th component pattern, the $S c_{k} y(2 \theta)_{k}^{\mathrm{S}}$ is fitted and $Y_{k}^{\mathrm{BP}}$ is output. If we predetermine the magnitude of $R_{k}$, the $Y_{k}$ can be derived from $Y_{k}^{\mathrm{BP}}$ with Eq. (14), and it can be used in Eq. (9) together with other $S_{k}$ and/or $Y_{k}$. Two experimental techniques for determining values of $R_{k}$ are given in Toraya (2019).

When the type- $\mathrm{C}_{2}$ function is assigned to all components in a mixture, the substitution of Eq. (14) into Eq. (9) gives

$$
w_{k}=\frac{a_{k} Y_{k}^{\mathrm{BP}}}{1+R_{k}}\left(\sum_{k^{\prime}=1}^{K} \frac{a_{k^{\prime}} Y_{k^{\prime}}^{\mathrm{BP}}}{1+R_{k^{\prime}}}\right)^{-1}
$$

When the following approximation does hold

$$
R_{1} \cong R_{2} \cong R_{3} \cong \cdots \cong R_{K}
$$

Equation (15) becomes

$$
w_{k} \cong a_{k} Y_{k}^{\mathrm{BP}}\left(\sum_{k^{\prime}=1}^{K} a_{k^{\prime}} Y_{k^{\prime}}^{\mathrm{BP}}\right)^{-1}
$$

Equation (17) has the same form as that of Eq. (9). In this case, the weight fraction $w_{k}$ can be derived by using the intensity datasets $\left\{Y_{k}^{\mathrm{BP}}\right\}$ of non-BG subtracted powder patterns, and the parameter $R_{k}$ is not required. For a mixture consisting of component materials with similar chemical compositions, the relation by Eq. (16) generally holds, and the type- $\mathrm{C}_{2}$ function gives very accurate results of quantification as will be shown later.

Important thing to be noted here is that four types of fitting functions given in Table I can arbitrarily be combined and be fitted simultaneously in WPPF for a mixture pattern, and an example will be shown later. The other thing is that the type-A function has the greatest degree of freedom in WPPF. The type-A function is the same as that used in Pawley refinement, and individual integrated intensities as well as unit-cell parameters, profile width, and shape parameters can be refined. When single-phase observed powder diffraction patterns are used as the type- $\mathrm{C} / \mathrm{C}_{2}$ function, they should be measured under the same instrumental setup as that for measuring the target mixture patterns in order to reduce the model bias. In this sense, the type-C and type- $\mathrm{C}_{2}$ functions are less flexible compared with the type-A and B functions. They are, however, stable in the least-squares fitting and suitable for modeling complicated diffraction patterns in WPPF.

Regarding the effect of the preferred orientation on individual intensities, the DD method, utilizing the total sums of scattered/diffracted intensities is much less influenced than the single-peak intensity methods. The intensity data are, however, not corrected for the preferred orientation at present. The correction for the preferred orientation effect as well as microabsorption effect are issues to be studied in the future.

\section{Introduction of the normalized fitting function}

Since Eq. (6) has been derived by summing/integrating all scattered/diffracted intensities in the reciprocal space, the definition range of $2 \theta$ for the corresponding total sums of observed intensities, defined by Eqs. (5) and (12), should also be extended to the maximum high-angle limit. In QPA using Eqs. (9) and (17), the relative intensity ratios of $S_{1}$ : $S_{2}: S_{3}: \cdots: S_{K}$ determine the weight fractions. When one component in a mixture has relatively strong peaks in the highangle region, compared with the other components, the relative intensity ratios will be varied with whether these peaks are included or not in the range $\left[2 \theta_{\mathrm{L}}, 2 \theta_{\mathrm{H}}\right]$. The termination in summing/integrating observed intensities is, therefore, one of the possible sources of error in the derived weight fractions. In order to suppress the termination errors, the normalized fitting functions have been introduced (Toraya, 2020). In the case, for example, of type-C function, the normalized type-C function, denoted by $y(2 \theta)_{k}^{\mathrm{N}}$, can simply be defined by $y(2 \theta)_{k}^{\mathrm{N}}=y(2 \theta)_{k}^{\prime} / Y_{0 k}$. Here, $Y_{0 k}$ is equivalent to $Y_{k}$ defined by Eq. (12). Then, the integration of $y(2 \theta)_{k}^{\mathrm{N}}$ always gives unity, that is, $\int_{2 \theta_{\mathrm{L}}}^{2 \theta_{\mathrm{H}}} y(2 \theta)_{k}^{\mathrm{N}} G(2 \theta) \mathrm{d}(2 \theta)=1$. In WPPF using the normalized type-C function, $S c_{k}^{\mathrm{N}} y(2 \theta)_{k}^{\mathrm{N}}$ is fitted, where $S c_{k}^{\mathrm{N}}$ is the adjustable scale parameter for the normalized fitting function. Then, $Y_{k}$, defined by Eq. (12), but for the normalized fitting function, is given by $Y_{k}=S c_{k}^{\mathrm{N}}$.

The advantages of introducing the normalized fitting function are that the magnitude of refined scale parameter, $S c_{k}^{\mathrm{N}}$, is much less sensitive to the termination of the $2 \theta$-range in WPPF. If we define the normalized fitting function by extending the $\left[2 \theta_{\mathrm{L}}, 2 \theta_{\mathrm{H}}\right]$ to the high-angle limit such as $100^{\circ}$ or $120^{\circ}$ (for $\mathrm{CuK \alpha}$ radiation), the $2 \theta$-range in WPPF for target mixture patterns can be much shortened to, say, $60^{\circ}$. Therefore, we can save the time spent for intensity data collection without losing the accuracy in derived weight fractions (Toraya, 2020).

\section{E. Calculation and some properties of $a_{k}$}

We can easily calculate $a_{k}$ values from the chemical composition data using a periodic table and a pocket calculator. Here, two examples are given. One is $\alpha$-quartz with the chemical formula of $\mathrm{SiO}_{2}$. In this case, $M_{k}$ and $\sum n_{i}^{2}$ are given by $M_{k}=28.086+2 \times 15.999=60.084 \mathrm{~g} \mathrm{~mol}^{-1}$ and $\sum n_{i}^{2}=$ $14^{2}+2 \times 8^{2}=324$, respectively. Thus, $a_{k}=M_{k} / \sum n_{i}^{2}=$ 0.18544 . The other example is a solid solution with the chemical formula, for example, $\mathrm{Mg}_{1.6} \mathrm{Fe}_{0.4} \mathrm{SiO}_{4} . M_{k}$ and $\sum n_{i}^{2}$ are given by $M_{k}=1.6 \times 24.312+0.4 \times 55.847+28 . \quad 086+4 \times$ $15.999=153.320 \mathrm{~g} \mathrm{~mol}^{-1}$ and $\sum n_{i}^{2}=1.6 \times 12^{2}+0.4 \times$ $26^{2}+14^{2}+4 \times 8^{2}=952.8$. Thus, $a_{k}=0.16092$. For fractional atoms like $\mathrm{Mg}_{x}$, the $n_{i}^{2}$ should be counted as $x \times 12^{2}$ 
TABLE II. A comparison of $a_{k}$ values for series of magnesium silicate hydrates and hydrocarbons with similar chemical compositions (Toraya, 2017).

\begin{tabular}{llll}
\hline \hline Chemical formula & \multicolumn{1}{c}{$a_{k}$} & Chemical formula & $a_{k}$ \\
\hline $\mathrm{Mg}_{3}\left(\mathrm{SiO}_{4}\right)(\mathrm{OH})_{2}$ & 0.19028 & $\mathrm{C}_{10} \mathrm{H}_{8}$ & 0.3483 \\
$\mathrm{Mg}_{5}\left(\mathrm{SiO}_{4}\right)_{2}(\mathrm{OH})_{2}$ & 0.19023 & $\mathrm{C}_{14} \mathrm{H}_{10}$ & 0.3468 \\
$\mathrm{Mg}_{7}\left(\mathrm{SiO}_{4}\right)_{3}(\mathrm{OH})_{2}$ & 0.19020 & $\mathrm{C}_{18} \mathrm{H}_{12}$ & 0.3459 \\
$\mathrm{Mg}_{9}\left(\mathrm{SiO}_{4}\right)_{4}(\mathrm{OH})_{2}$ & 0.19019 & $\mathrm{C}_{22} \mathrm{H}_{14}$ & 0.3454 \\
Average & $0.19022(4)$ & Average & $0.3466(11)$ \\
\hline \hline
\end{tabular}

Values at the bottom line represent the averages of individual $a_{k}$ values and their standard deviations in parentheses.

but not as $(x \times 12)^{2}$. In the average structure, one crystallographic site is shared like $\mathrm{Mg}_{x} \mathrm{Fe}_{1-x}$. In real space, however, atoms at individual sites in a whole crystal scatter the $n_{i}^{2}$ of either $12^{2}$ or $26^{2}$ in an abundance ratio of $x: 1-x$.

To understand some properties of $a_{k}$ may be useful when the chemical composition data of target materials are more or less indefinite. In the first, the $a_{k}$ has the relationship represented by $a_{k}^{-1} \approx A_{k}^{\text {av }} / D$, where $A_{k}^{\text {av }}$ is the average atomic weight of atoms in the chemical formula unit $\left(A_{k}^{\mathrm{av}}=M_{k} / N_{k}^{\mathrm{A}}\right), D$ is a ratio of atomic weight to atomic number, and $D \approx 2(D=$ 2.006 in the case of Si) (Toraya, 2017, 2018). Table II gives the values of $a_{k}$ for two series of compounds (Toraya, 2017). In the case of hydrated magnesium silicate (HMS), the standard deviation of the average $a_{k}$ value is just $0.02 \%$. These results mean that materials with similar chemical compositions give the $a_{k}$ 's of almost the same magnitude. In the case of polymorph or polytypes, the $a_{k}$ 's are equivalent, and we can derive individual weight fractions, $w_{k}$, by the IC formula without using the $a_{k}$. When four compounds of HMS in Table II are evenly weighed, mixed and quantified by using the average $a_{k}$ of 0.19022 instead of individual $a_{k}$ values, associated error in $w_{k}$ is just $0.02 \mathrm{wt} \%$ (Toraya, 2017).

As well known, chemicals compositions of natural products are complicated due to various degrees of cation substitution like $\mathrm{Si} \leftrightarrow \mathrm{Al}, \mathrm{Mg} \leftrightarrow \mathrm{Fe}$, as well as inclusions of trace elements, such as $\mathrm{Li}, \mathrm{Ti}, \mathrm{Cr}, \mathrm{Mn}, \mathrm{Ni}, \mathrm{Ca}, \mathrm{Cs}$, and $\mathrm{Ba}$. The chemical composition data of rock-forming minerals are reported from all over the world (Deer et al., 1971, 1976), and we can calculate the average of $a_{k}$ value, denoted by $a_{k}^{\text {av }}$, and its standard deviation, $\sigma\left(a_{k}^{\mathrm{av}}\right)$, for each rock-forming mineral. As a practical example, a simulated QPA result is given for weathered granite, consisting of $\alpha$-quart $\left(\mathrm{SiO}_{2}\right)$, orthoclase $\left(\mathrm{KSi}_{3} \mathrm{AlO}_{8}\right)$, albite $\left(\mathrm{NaSi}_{3} \mathrm{AlO}_{8}\right)$, and biotite $\left[\mathrm{K}(\mathrm{Fe}, \mathrm{Mg})_{3} \mathrm{Si}_{3} \mathrm{AlO}_{10}(\mathrm{OH})_{2}\right]$ in weight ratios of 48:37:11:4. Errors in $w_{k}$, represented by $\Delta w_{k}$, from assumed weight fractions were generated by varying $a_{k}$ by $a_{k}^{\mathrm{av}} \pm \sigma\left(a_{k}^{\mathrm{av}}\right)$. The average values of $\left|\Delta w_{k}\right|$ were $0.33,0.51,0.10$, and $0.16 \mathrm{wt} \%$ for respective minerals (a grand average of $0.27 \mathrm{wt} \%$ ). These errors can also be estimated with the error estimation formula [Eq. (11) in Toraya (2017)], and the magnitudes of estimated errors were $0.37,0.48,0.11$, and $0.15 \mathrm{wt} \%$ ( $0.28 \mathrm{wt} \%)$, being in a good agreement with those in simulated QPA. These results mean that errors associated with possible variations in $a_{k}$ value will be in the order of $<0.5 \mathrm{wt} \%$, and they may be less for minerals from local mines with accumulated chemical analysis data.

When unknown material was found as one of the components in a mixture, its chemical composition can be estimated, as the first approximation, by the batch chemical composition of the sample. More accurate procedure for estimating $a_{k}$ and $w_{k}$ of the unknown material using the iterative procedure is described in Toraya (2017).

\section{EXPERIMENTAL AND DATA ANALYSIS}

Intensity data collections can be conducted with an ordinary type powder diffractometer based on the BraggBrentano geometry. The $G_{j}$ in Eq. (5) or $G(2 \theta)$ in Eq. (12) are needed to be modified accordingly, when the monochromator was used on the incident- or diffracted-beam sides, the other types of diffractometer were used, or the different scan-mode such as the asymmetric diffraction was employed. As was described above, the constant optical setting should be kept in measuring powder diffraction patterns of both target mixtures and single-phase components to be used as the type- $C$ and/or the type- $C_{2}$ functions. When the type- $C / C_{2}$ functions were used, the shift of a whole pattern, $y(2 \theta)_{k}^{\prime}$ or $y(2 \theta)_{k}^{\mathrm{S}}$, along the $2 \theta$-axis, associated primarily with the shift in the height of a specimen surface, can be corrected by adjusting the parameter, $\Delta 2 \theta_{k}$ (Toraya, 2018) $\left[\delta_{2 \theta k}\right.$ is a different symbol (Toraya, 2019, 2020)]. Regarding the $2 \theta$-range for scanning, $\left[2 \theta_{\mathrm{L}}, 2 \theta_{\mathrm{H}}\right]$, the $2 \theta_{\mathrm{L}}$ should include the reflection at the lowest angle. The $2 \theta_{\mathrm{H}}$ should be extended to $100^{\circ}$ or $120^{\circ}$ (for the $\mathrm{Cu} K \alpha$ radiation), depending on the accuracy required. When the normalized fitting functions are used, the $2 \theta_{\mathrm{H}}$ for target mixtures can be lowered to $60^{\circ}$ or $70^{\circ}$.

A computer program, WPPF4.0 (version 4.00), written in Fortran 90 for the WPPD method (Toraya, 1986) has been used for data analysis, conducted by the author himself. Some software suites are commercially available. Although terms of type-A, B, C, and $\mathrm{C}_{2}$ functions were not used before, these four types of the fitting functions have already been equipped and used as they are classified in Table I. Therefore, existing computer programs for WPPD, Rietveld refinement, full-pattern fitting, etc. can also be used with a small modification or by adding a subprogram for calculating $S_{k}$ [Eq. (5)], $Y_{k}$ [Eq. (12)], and $Y_{k}^{\mathrm{BP}}$ [Eq. (13)] together with $a_{k}$ values. The details of experimental and data analysis conditions for analyzed results given below will be found in respective references cited.

\section{RESULTS AND DISCUSSION}

In the followings, five examples of applications of the DD method are presented.

\section{A. Combined use of type-A, B, and C functions}

A first example is the QPA of an artificial ternary mixture consisting of $\alpha$-quartz $\left(\mathrm{SiO}_{2}\right)$, albite $\left(\mathrm{NaAlSi}_{3} \mathrm{O}_{8}\right)$, and kaolinite $\left(\mathrm{Al}_{2} \mathrm{Si}_{2} \mathrm{O}_{5}(\mathrm{OH})_{4}\right)$ in weight ratios of 5:4:1, which simulate weathered granites used as raw materials in the ceramics industry. Mixtures in weight ratios of 1:1:1 were also quantified (Toraya, 2018). $\alpha$-quartz with a relatively small unit cell belongs to the trigonal symmetry, and it gives well-resolved peaks sparsely distributed in its diffraction pattern. On the other hand, albite belongs to the triclinic system, and it gives a lot of reflections heavily overlapping in a whole $2 \theta$-range. As well known, kaolinite is a clay mineral, and it exhibits diffuse scattering caused by the stacking disorder. 
TABLE III. Results of the QPA of two mixtures in weight ratios of 5:4:1 and $1: 1: 1$.

\begin{tabular}{lccccc}
\hline \hline Component & Scan & $\alpha$-quartz & Albite & Kaolinite & RMSE \\
\hline$w^{\text {weigh }}$ & & 50.00 & 39.97 & 10.03 & \\
$5: 4: 1$ & 1st & -0.54 & -0.02 & 0.57 & 0.46 \\
& 2nd & 0.78 & -0.78 & 0.00 & 0.63 \\
& 3rd & 0.34 & -0.81 & 0.47 & 0.57 \\
$w^{\text {weigh }}$ & & 33.33 & 33.33 & 34.34 & \\
$1: 1: 1$ & 1st & 0.036 & -2.11 & 1.76 & 1.60 \\
& 2nd & -0.41 & -1.85 & 2.25 & 1.70 \\
& 3rd & 0.32 & -2.48 & 2.16 & 1.91 \\
\hline \hline
\end{tabular}

Numbers for each scan represent $\Delta w_{k}=w_{k}-w_{k}^{\text {weigh }}$ (wt $\%$ ), where $w_{k}^{\text {weigh }}$ represents the weighed value of each component in sample preparation. The root-mean-square errors (RMSE) of $\Delta w_{k}$ for three components are given on the right-hand column (Toraya, 2018).

When these materials exist as a mixture, a straightforward application of the Pawley-based WPPD method is hard to decompose the mixture pattern into individual Bragg components with sufficient accuracy.

For separating the mixture pattern into individual component patterns, a strategy taken for this mixture sample was to assign the type- $\mathrm{A}, \mathrm{B}$, and $\mathrm{C}$ functions to $\alpha$-quartz, albite, and kaolinite, respectively. Before applying these functions to the mixture pattern in WPPF, a single-phase diffraction pattern of albite was first decomposed to obtain an intensity dataset $\left\{I_{k j}^{\prime}\right\}$. In the case that the single-phase sample is not available, an alternative way may be to derive the $\left\{I_{k j}^{\prime}\right\}$ by calculation using crystal structure parameters. Secondly, the BG of a single-phase diffraction pattern of kaolinite was subtracted to obtain $y(2 \theta)_{k}^{\prime}$. In WPPF for the mixture pattern, $I_{j k}$ parameters of $\alpha$-quartz were refined independently together with the scale parameters, $S c_{k}$, for albite and kaolinite patterns. The unit-cell parameters of $\alpha$-quartz and albite were also refined together with the other parameters such as profile width and peak-shift correction.

For testing the reproducibility in this example, intensity measurements of the two mixture samples, as well as the
TABLE IV. $\quad w_{k}^{\text {weigh }}$ and $\Delta w_{k}$ (in wt $\%$ ) for $\alpha$ - and $\gamma-\mathrm{Al}_{2} \mathrm{O}_{3}$ mixtures with five different weight ratios.

\begin{tabular}{lcccccc}
\hline \hline wt. ratio & $95: 5$ & $75: 25$ & $50: 50$ & $25: 75$ & $5: 95$ & $\left|\Delta w_{k}\right|_{\mathrm{av}}$ \\
\hline$w_{k}^{\text {weigh }}$ & 5.01 & 25.05 & 50.04 & 75.00 & 95.01 & \\
$\Delta w_{k}$ & -0.04 & -0.10 & -0.00 & -0.05 & -0.06 & 0.05 \\
\hline \hline
\end{tabular}

Data are given only for $\gamma-\mathrm{Al}_{2} \mathrm{O}_{3}$ since those for $\alpha-\mathrm{Al}_{2} \mathrm{O}_{3}$ can be obtained by $1-w_{k}^{\text {weigh }}$ and $-\Delta w_{k}$.

three component materials, were repeated three times for each sample, repacked into a specimen holder prior to each scan. Before preparing the second mixture (1:1:1), a powder of kaolinite was additionally ground in an agate mortar in order to examine the influence of the degree of preferred orientation.

Table III gives the results of QPA. The root-mean-square error (RMSE) of $\Delta w_{k}$ was in the range $0.5-0.6 \mathrm{wt} \%$ for the $5: 4: 1$ mixture, while it was in the range 1.6-1.9 $\mathrm{wt} \%$ for the 1:1:1 mixture. The increase of the RMSE by $\sim 1.2 \mathrm{wt} \%$ was ascribed to the different degrees of the preferred orientation of kaolinite particles between the two mixtures: kaolinite powder was additionally ground in preparing the second mixture, while the diffraction pattern of a kaolinite before additional grinding was used as the type-C function in the QPA of both mixtures. These results give a simple lesson that the diffraction pattern used for the type- $\mathrm{C}$ function should be close to that in the target mixture pattern.

\section{B. QPA using type- $C_{2}$ function \\ 1. QPA of $\alpha-\mathrm{Al}_{2} \mathrm{O}_{3}+\gamma-\mathrm{Al}_{2} \mathrm{O}_{3}$ binary mixtures}

The second example is the use of the type- $\mathrm{C}_{2}$ function, applied to binary mixtures of $\alpha$ - and $\gamma-\mathrm{Al}_{2} \mathrm{O}_{3}$ in five different weight ratios (Table IV; Toraya, 2019). $\alpha-\mathrm{Al}_{2} \mathrm{O}_{3}$ is chemically and thermally stable, and it is often used as a standard reference material, giving well-resolved peaks in a full $2 \theta$-range. On the other hand, $\gamma-\mathrm{Al}_{2} \mathrm{O}_{3}$ has the structure of a defect cubic spinel type, and it gives broadened profiles and diffuse

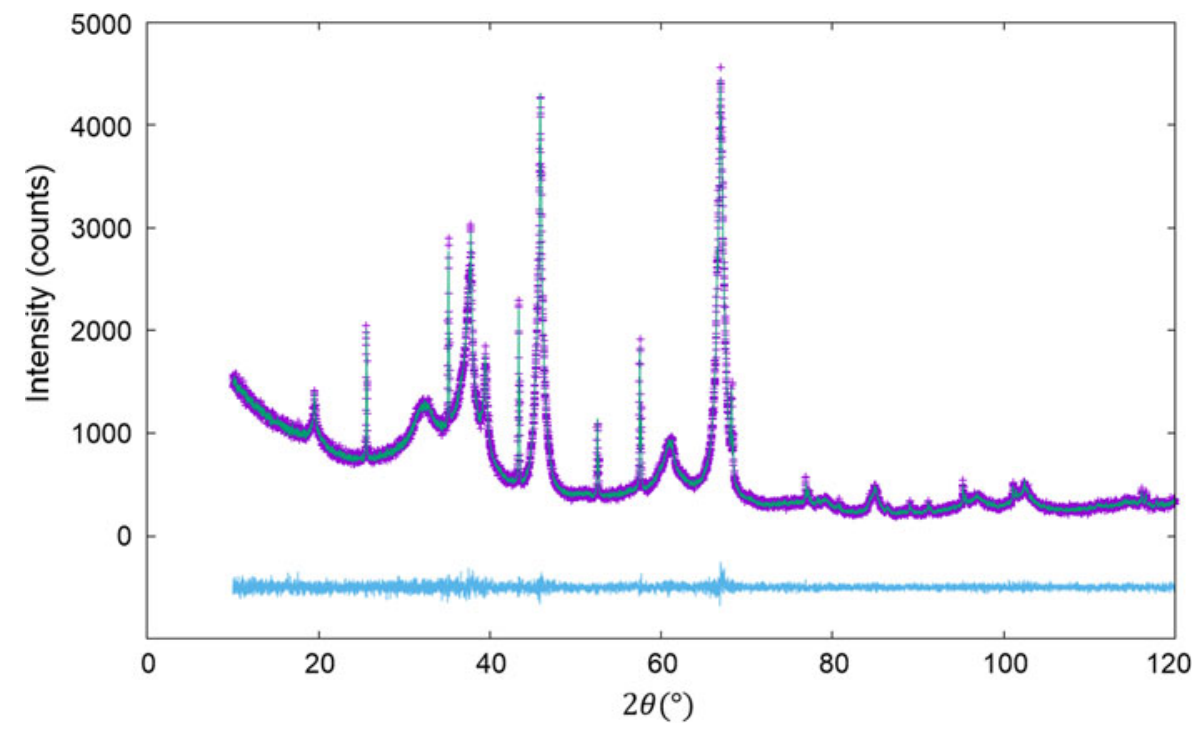

Figure 1. WPPF result for the diffraction pattern of $\alpha$ - and $\gamma-\mathrm{Al}_{2} \mathrm{O}_{3}$ mixture with a weight ratio of 5:95 (Toraya, 2019). The observed and calculated intensities are represented by plus symbols and solid lines, respectively. The plot at the bottom of the diagram represents the differences between the two intensities on the same scale. 
TABLE V. $w_{k}^{\text {weigh }}$ and $\Delta w_{k}$ (in wt $\%$ ) for $\alpha$-quartz $\left(\mathrm{SiO}_{2}\right)$ and glass-SiO mixtures with four different weight ratios.

\begin{tabular}{lccccc}
\hline \hline wt. ratio & $80: 20$ & $60: 40$ & $40: 60$ & $20: 80$ & $\left|\Delta w_{k}\right|_{\mathrm{av}}$ \\
\hline$w_{k}^{\text {weigh }}$ & 19.68 & 40.35 & 60.01 & 80.17 & \\
$\Delta w_{k}$ & 0.6 & 0.2 & 0.3 & -0.5 & 0.4 \\
& 0.4 & -0.2 & -0.4 & -0.7 & 0.4 \\
\hline \hline
\end{tabular}

Data are given only for glass components. $\left|\Delta w_{k}\right|_{\text {av }}$ represents the average of four $\left|\Delta w_{k}\right|$ (Toraya, 2019). $\Delta w_{k}$ values on the first line were obtained without the correction by the BG function and those on the second line by adjusting 8 BG parameters.

scattering as will be shown in Figure 1. Neither structure modeling from crystal structure parameters nor the pattern decomposition into Bragg components was difficult. Moreover, the diffuse scattering spread over the full $2 \theta$-range made difficult to determine the accurate $\mathrm{BG}$ height. The type- $\mathrm{C}_{2}$ function is best suited for such a sample.

Both $\alpha$ - and $\gamma-\mathrm{Al}_{2} \mathrm{O}_{3}$ have the same chemical composition, and the relation $R_{\alpha} \cong R_{\gamma}$ [Eq. (16)] was considered to hold. Then, single-phase powder diffraction patterns of $\alpha$ and $\gamma-\mathrm{Al}_{2} \mathrm{O}_{3}$ were used as the type- $\mathrm{C}_{2}$ function. In WPPF to the mixture patterns, $S c_{k}$ and $\delta_{2 \theta k}$ for both components were refined together with the parameters in the $\mathrm{BG}$ function.
TABLE VI. $\quad w_{k}^{\text {weigh }}$ and $\Delta w_{k}$ (in wt $\%$ ) for corn starch in corn-potato starch mixtures.

\begin{tabular}{lcccccc}
\hline \hline wt. ratio & $95: 05$ & $70: 30$ & $50: 50$ & $30: 70$ & $5: 95$ & $\left|\Delta w_{k}\right|_{\mathrm{av}}$ \\
\hline$w_{k}^{\text {weigh }}$ & 94.9 & 70.0 & 50.0 & 30.0 & 5.0 & \\
$\Delta w_{k}$ & -0.4 & -1.9 & -2.2 & 0.8 & 0.5 & 1.2 \\
\hline \hline
\end{tabular}

Data are given only for corn starch. $\left|\Delta w_{k}\right|_{\mathrm{av}}$ represents the average of five | $\Delta w_{k}$.

Figure 1 shows a WPPF result for the mixture in weight ratio of 5:95. Table IV gives QPA results. An average value of $\Delta w_{k}$ for five mixtures was just $0.05 \mathrm{wt} \%$. In the case of mixture in weight ratio of 95:5, it was hard to see a presence of $\gamma-\mathrm{Al}_{2} \mathrm{O}_{3}$ in the observed pattern. Nevertheless, $\gamma-\mathrm{Al}_{2} \mathrm{O}_{3}$ was accurately quantified. Simple adjustments of two single-phase diffraction patterns realize the accurate quantification of mixtures with complicated diffraction patterns.

\section{QPA of amorphous component}

QPA procedure used in the second example can directly be applied to mixture patterns containing an amorphous component. The third example is binary mixtures of $\alpha$-quartz

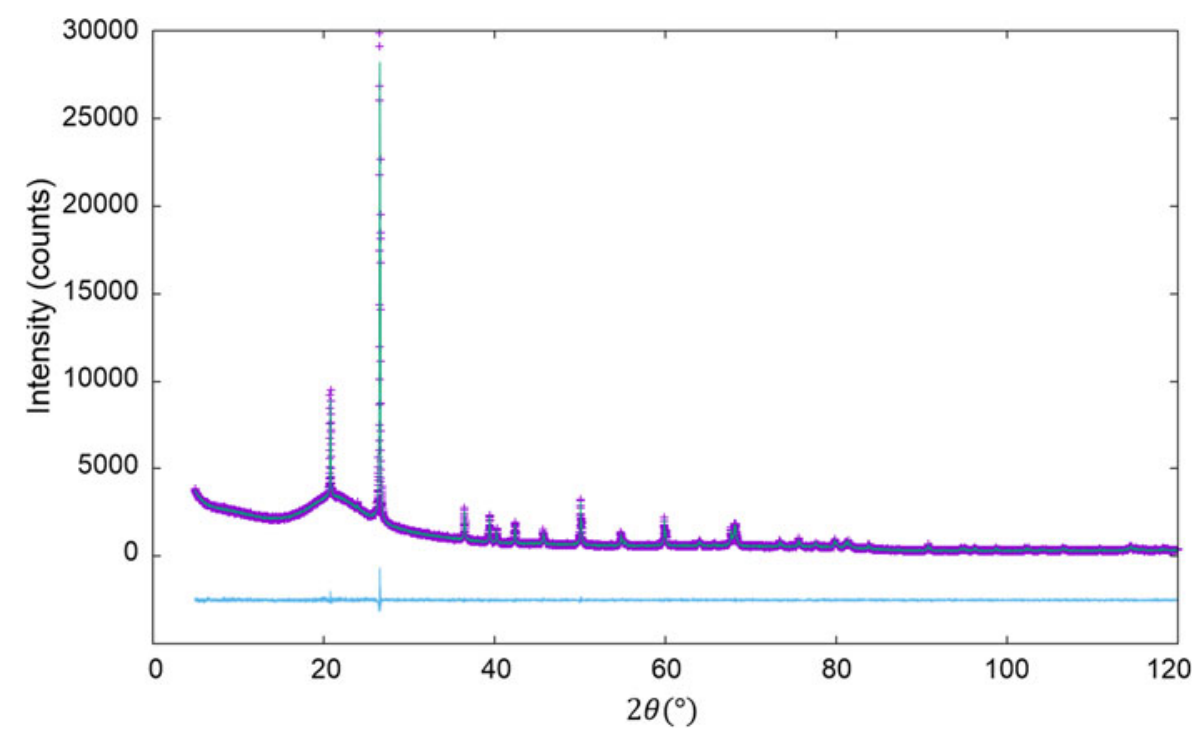

Figure 2. WPPF result for the diffraction pattern of $\alpha$-quartz $\left(\mathrm{SiO}_{2}\right)$ and glass- $\mathrm{SiO}_{2}$ mixture in a weight ratio of 2:8 (Toraya, 2019). Data are plotted as in Figure 1.

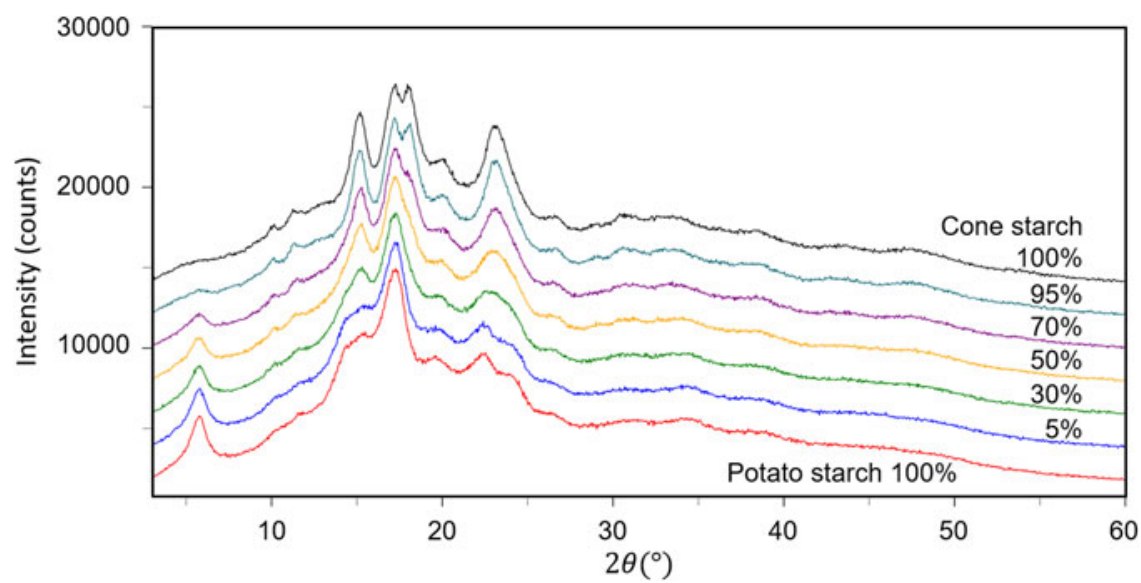

Figure 3. Powder diffraction patterns of potato starch, corn starch, and their mixtures in weight ratios indicated in the diagram. 
TABLE VII. $\quad w_{k}^{\text {weigh }}$ and $\left|\Delta w_{k}\right|_{\mathrm{av}}$ (in wt $\%$ ) for two series of mixtures SA and GS.

\begin{tabular}{llllll}
\hline \hline Mixture & SA030 & SA020 & SA010 & SA005 & SA003 \\
$w_{k}^{\text {weigh }}$ & 0.324 & 0.214 & 0.107 & 0.054 & 0.031 \\
$\left|\Delta w_{k}\right|_{\mathrm{av}}$ & $0.002(1)$ & $0.014(1)$ & $0.009(1)$ & $0.014(1)$ & $0.016(3)$ \\
Mixture & GS030 & GS020 & GS010 & GS005 & GS002 \\
$w_{k}^{\text {weigh }}$ & 0.323 & 0.211 & 0.100 & 0.052 & 0.021 \\
$\left|\Delta w_{k}\right|_{\mathrm{av}}$ & $0.059(2)$ & $0.029(1)$ & $0.026(1)$ & $0.024(1)$ & $0.017(3)$ \\
\hline
\end{tabular}

Data are given only for minor phases, A and S. Numbers in parentheses for I $\Delta w_{k} l_{\text {av }}$ represent the standard deviations for respective averages (Toraya, 2020).

$\left(\mathrm{SiO}_{2}\right)$ and glass-SiO${ }_{2}$ in four different weight ratios (Table V). As in the previous example, the diffraction pattern of single-phase $\alpha$-quartz and the halo pattern of glass- $\mathrm{SiO}_{2}$ were separately measured, and both patterns were used as the type- $\mathrm{C}_{2}$ function without subtracting their BG. Figure 2 shows a WPPF result for a mixture in weight ratio of 2:8. When the type-C function, instead of the type- $\mathrm{C}_{2}$, was assigned to the glass- $\mathrm{SiO}_{2}$ in WPPF for the same mixtures, the scale parameter for the glass halo pattern was interacted with the parameters in the $\mathrm{BG}$ function, and errors were increased with decreasing the content of glass- $\mathrm{SiO}_{2}$ (Toraya and Omote, 2019). The type- $\mathrm{C}_{2}$ function can avoid this parameter interaction, and it contributed to obtain accurate QPA results as indicated in Table $\mathrm{V} . \Delta w_{k}$ values on the two lines in Table $\mathrm{V}$ were obtained with and without using the $\mathrm{BG}$ function for modeling the $\mathrm{BG}$. These results indicate that the sum of the BG intensities from both component patterns, rep-

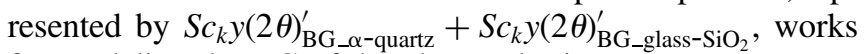
for modeling the $\mathrm{BG}$ of the observed mixture patterns.

\section{QPA of starch}

The fourth example is the QPA of binary mixtures consisting of potato starch $\left[\left(\mathrm{C}_{6} \mathrm{H}_{10} \mathrm{O}_{5}\right)_{n}\right]$ and corn starch $\left(\mathrm{C}_{27} \mathrm{H}_{48} \mathrm{O}_{20}\right)$ artificially mixed in five different weight ratios (Table VI). Figure 3 shows the powder diffraction patterns of the mixtures together with those of single-phase potato and corn starches. Although the two single-phase diffraction patterns indicate clear differences at some strong peaks while they are very similar to each other in other angular regions, and the mixture
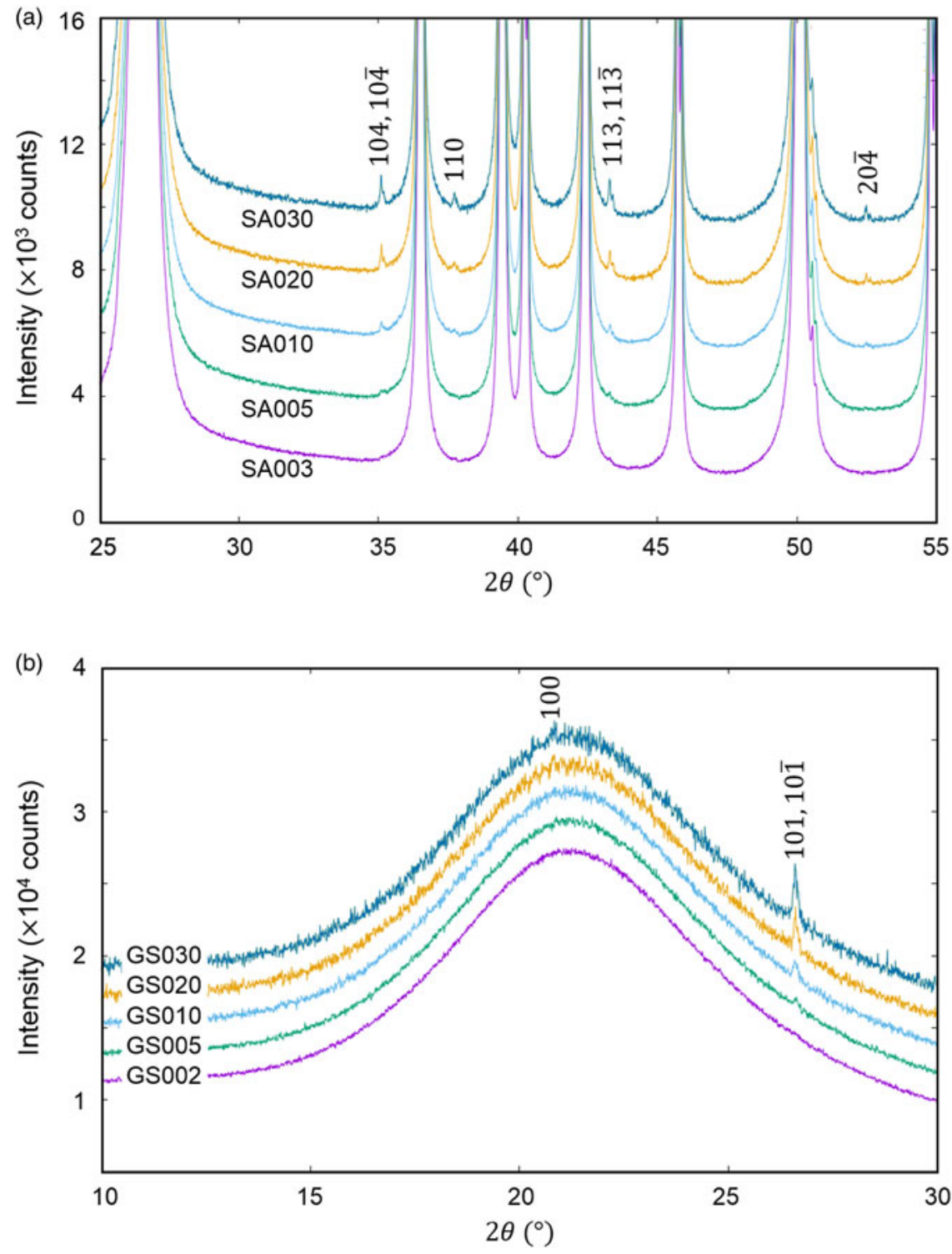

Figure 4. Observed powder diffraction patterns (parts) of (a) mixtures SA and (b) GS. Individual patterns are vertically shifted from each other at equal intervals of 2000 counts. Indices in the diagrams represent reflections from (a) $\alpha-\mathrm{Al}_{2} \mathrm{O}_{3}$ and (b) $\alpha-\mathrm{SiO}_{2}$ (Toraya, 2020). 
patterns gradually change between the two ends. The QPA of such materials has been very difficult because the RIR values are not available for these materials in the database. Structure-based Rietveld QPA could also be infeasible. In the present case, the single-phase diffraction patterns of potato and corn starches were used as the type- $\mathrm{C}_{2}$ functions, and they were fitted in WPPF to the mixture patterns. The results of QPA are given in Table VI, giving errors of just $1.2 \mathrm{wt} \%$ in their average.

\section{QPA of crystalline phase present in a very small amount in a mixture}

The QPA of crystalline phases present in a very small amount, say $<0.5 \mathrm{wt} \%$, in mixtures is a very important issue in quality control of industrial products as well as in the research and development. The last example is the QPA of mixtures of (a) $\alpha-\mathrm{SiO}_{2}+\alpha-\mathrm{Al}_{2} \mathrm{O}_{3}$ (SA) and (b) glass- $\mathrm{SiO}_{2}+$ $\alpha-\mathrm{SiO}_{2}$ (GS) in five different weight ratios (Table VII), where the second components represent minor phases (Toraya, 2020). As shown in Figure 4, the presence of crystalline phases as minor components can be discerned as tiny peaks in both mixtures.

In WPPF, the normalized type- $\mathrm{C}_{2}$ function was assigned to all component in SA and GS mixtures. In Table VII, I $\left.\Delta w_{k}\right|_{\text {av }}$ represents the average of five $\left|\Delta w_{k}\right|$, individual values of which were results of WPPF in the range of $2 \theta_{\mathrm{H}}=60^{\circ}$, $70^{\circ}, 80^{\circ}, 90^{\circ}$, and $100^{\circ}\left(2 \theta_{\mathrm{L}}=15^{\circ}\right)$. As clearly be shown in the very small standard deviations of $0.001-0.003 \mathrm{wt} \%$ for the $\left|\Delta w_{k}\right|_{\mathrm{av}}$, the scale parameters $\left(S c_{k}^{\mathrm{N}}\right)$ were almost unvaried against the change of $2 \theta$-range in WPPF. In this case, QPA could be conducted in the accuracy of $0.01-0.03 \mathrm{wt} \%$ for the mixtures containing component materials in wt $\%$ of $0.02-0.4$.

\section{SUMMARY}

Theoretical background of the DD method and examples of applications have been described in this report. Some descriptions with many equations would give an impression that the DD method is a complicated technique. The DD method itself is, however, very simple, and it can simply be expressed by the IC formula [Eq. (9)]. It is based on a principle that the weight proportion for a component material can be given by dividing the total sum of scattered/diffracted intensities by the scattered intensity per unit weight represented by the symbol, $a_{k}^{-1}$. Moreover, the magnitude of the $a_{k}^{-1}$ can be calculated only with the chemical composition data of that component material. Therefore, as indicated with several examples of applications given in this report, the DD method can be applied to the variety of materials from highly crystalline state to noncrystalline state just with the IC formula. Only a technical problem is how to separate the powder diffraction pattern of a target mixture into individual component patterns.

Various existing techniques can be used for tackling the problem. In the present report, four types of the fitting functions for WPPF are described. What we need as final outputs as the observed data in powder data analysis are $S_{k}, Y_{k}$, and/or $Y_{k}^{\mathrm{BP}}$. For this purpose, for example, even intensity datasets in d-I data in the PDF or instead the corresponding observed peak intensities can be used for calculating $S_{k}$ by Eq. (5). The relative weight ratios can be output immediately after the phase identification if a rough estimate of relative weight ratios suffices. As another way, currently used computer programs can also be used with small modification. For example, if we add the type-C and the type- $\mathrm{C}_{2}$ functions for modeling the amorphous components to a computer program for Rietveld refinement, the weight fractions can directly be output not only for crystalline phases but also for noncrystalline phase without doping the standard reference material nor conducting preliminary experiment. Individual techniques for separating the mixture patterns look sometimes complicated. In other words, however, there are various ways to solve a simple problem of just obtaining $S_{k}, Y_{k}$, and/or $Y_{k}^{\mathrm{BP}}$.

\section{ACKNOWLEDGEMENTS}

The author thanks Yukiko Namatame of Application Laboratory, Rigaku Corporation (RC). All intensity datasets used in a series of studies on the DD method were collected by her. QPA results for mixtures of starches were also provided by her.

Alexander, L. E. and Klug, H. P. (1948). "Basic aspects of X-ray absorption in quantitative diffraction analysis of powder mixtures," Anal. Chem. 20, 886-889.

Averbach, B. L. and Cohen, M. (1948). "X-ray determination of retained austenite by integrated intensities," Trans. AIME 176, 401-415.

Chipera, S. J. and Bish, D. L. (2002). "FULLPAT: a full-pattern quantitative analysis program for X-ray diffraction using measured and calculated patterns," J. Appl. Crystallogr. 35, 744-749.

Chung, F. H. (1974a). "Quantitative interpretation of X-ray diffraction patterns of mixtures: I. Matrix-flushing method for quantitative multicomponent analysis," J. Appl. Crystallogr. 7, 519-525.

Chung, F. H. (1974b). "Quantitative interpretation of X-ray diffraction patterns of mixtures: II. Adiabatic principle of X-ray diffraction analysis of mixtures," J. Appl. Crystallogr. 7, 526-531.

Deer, W. A., Howie, R. A., and Zussman, J. (1971). Rock-Forming Minerals, Vol. 4. (Longman, London).

Deer, W. A., Howie, R. A., and Zussman, J. (1976). Rock-Forming Minerals, Vol. 3. (Longman, London).

Hubbard, C. R., Evans, E. H., and Smith, D. K. (1976). "The reference intensity ratio, $I / I_{\mathrm{c}}$, for computer simulated powder patterns," J. Appl. Crystallogr. 9, 169-174.

James, R. W. (1967). The Optical Principles of the Diffraction of X-Rays (Bell and Sons, London).

Le Bail, A., Duroy, H., and Fourquet, J. L. (1988). “Ab initio structure determination of $\mathrm{LiSbWO}_{6}$ by X-ray powder diffraction," Mater. Res. Bull. 23, 447-452.

Lennox, D. H. (1957). "Monochromatic diffraction-absorption technique for direct quantitative X-ray analysis,” Anal. Chem. 29, 766-770.

Parrish, W., Huang, T. C., and Ayer, G. L. (1976). "Profile fitting: a powerful method of computer X-ray instrumentation and analysis," Trans. Am. Cryst. Assoc. 12, 55-73.

Pawley, G. S. (1981). "Unit-cell refinement from powder diffraction scans," J. Appl. Crystallogr. 14, 357-361.

Rietveld, H. M. (1969). "A profile refinement method for nuclear and magnetic structures," J. Appl. Crystallogr. 2, 65-71.

Smith, D. K., Johnson Jr., G. G., Scheible, A., Wims, A. M., Johnson, J. L., and Ullmann, G. (1987). "Quantitative X-ray powder diffraction method using the full diffraction pattern," Powder Diffr. 2, 73-77.

Taupin, D. (1973). “Automatic peak determination in X-ray powder patterns," J. Appl. Crystallogr. 6, 266-273.

Toraya, H. (1986). "Whole-powder-pattern fitting without reference to a structural model: application to X-ray powder diffractometer data," J. Appl. Crystallogr. 19, 440-447. 
Toraya, H. (2016). "A new method for quantitative phase analysis using X-ray powder diffraction: direct derivation of weight fractions from observed integrated intensities and chemical compositions of individual phases," J. Appl. Crystallogr. 49, 1508-1516.

Toraya, H. (2017). "Quantitative phase analysis using observed integrated intensities and chemical composition data of individual crystalline phases: quantification of materials with indefinite chemical compositions," J. Appl. Crystallogr. 50, 820-829.

Toraya, H. (2018). "Direct derivation (DD) of weight fractions of individual crystalline phases from observed intensities and chemical composition data: incorporation of the DD method into the whole-powder-pattern fitting procedure," J. Appl. Crystallogr. 51, 446-455.

Toraya, H. (2019). "A practical approach to the direct-derivation method for QPA: use of observed powder patterns of individual components without background subtraction in whole-powder-pattern fitting," J. Appl. Crystallogr 51, 520-531.

Toraya, H. (2020). "Accurate and time-saving quantification of a component present in a very small amount in a mixture by the direct derivation method," J. Appl. Crystallogr. 53, 1225-1235.

Toraya, H. and Omote, K. (2019). "Quantitative phase analysis of amorphous components in mixtures by using the direct-derivation method," J. Appl. Crystallogr. 52, 13-22.

Toraya, H. and Tsusaka, S. (1995). "Quantitative phase analysis using the whole-powder-pattern decomposition method. I. Solution from knowledge of chemical compositions," J. Appl. Crystallogr. 28, 392-399.

Werner, P.-E., Salomé, S., and Malmros, G. (1979). "Quantitative analysis of multicomponent powders by full-profile refinement of Guinier-Hägg X-ray film data,” J. Appl. Crystallogr. 12, 107-109. 